Thus at step 7 we have

$$
\begin{array}{llll}
e_{12}-1=0 & e_{1}-2=0 & e_{2}-1=0 & e_{0}-3=0 \\
d_{12}+2=0 & d_{1}+3=0 & d_{2}+4=0 & d_{0}+4=0 .
\end{array}
$$

So there are 8 nonlinear equations in 20 unknowns. To make use of Bremermann's Algorithm, we have introduced new variables to obtain equations of the form $H_{i}=0$ so that each of the $H_{i}$ 's reduce to that of a polynomial of degree 2 . Thus we get 98 nonlinear equations in 110 unknowns. Some of the equations are given below:

$$
\begin{array}{ccc}
x_{1}-t_{3} t_{7}=0 ; & x_{2}-t_{13} t_{19}=0 ; & x_{3}-t_{3} t_{8}=0 ; \\
x_{4}-t_{7} t_{8}=0 ; & x_{5}-t_{14} t_{19}=0 ; & x_{6}-t_{4} t_{14}=0 ; \\
\because \because & \cdots: & \cdots \\
x_{88}-x_{33} x_{71}=0 ; & x_{89}-x_{34} x_{55}=0 ; & x_{90}-x_{34} x_{57}=0 \\
t_{1} x_{1}+t_{4} x_{2}-1=0 ; & \\
t_{1} t_{19}+t_{1} x_{3}+t_{1} x_{4}+t_{4} x_{5}+x_{3} x_{6}+t_{5} x_{2}+x_{1} x_{8} \\
-x_{1} x_{9}-x_{6} x_{10}-2=0 ; \\
::::::::::::::::::::::::::::::::::::::::::::
\end{array}
$$

Using a variant form of Bremermann's Algorithm [3] we have a set of solution for

$$
H=\sum_{i=1}^{98} H_{i}^{2}=0 .
$$

(In fact there are infinite many solutions): $t_{1}=1 ; t_{3}=1 ; t_{4}=-0.4$; $t_{5}=-0.6 ; \quad t_{6}=-0.8 ; \quad t_{7}=0.2 ; \quad t_{13}=1.195 ; \quad t_{14}=3.333 ; t_{17}=$ -0.1213 ; and $t_{2}=t_{8}=\cdots t_{20}=0$. With some additional arithmetic manipulations for exact fractions one can have $t_{1}=t_{3}=1$; $t_{4}=-\frac{2}{5} ; t_{5}=-\frac{3}{5} ; t_{6}=-\frac{4}{5} ; t_{7}=\frac{1}{5} ; t_{13}=\frac{6}{5} ; t_{14}=\frac{10}{3} ; t_{17}=-\frac{3}{25}$, and $t_{2}=t_{8}-\cdots t_{20}=0$.

The corresponding combinatorial realization is shown in Fig. 2. Since there is no delay free loop in the realization, this is also the circuit-theoretic realization.

\section{REFERENCES}

[1] S. Chakrabarti and S. K. Mitra, 'Correction to "Decision methods and realization of 2-D digital filters using minimum number of delay elements," IEEE Trans. Circuits Syst., vol. CAS-28, pp. 262-263, Mar. 1981

[2] S. J. Mason and H. J. Zimmerman, Electronic Circuits, Signals and Systems. New York: Wiley, 1960.

[3] H. Bremermann, "A method of unconstrained global optimization," Math. Biosci., vol. 9, pp. 1-15, 1970 .

\title{
Circuits and Systems Letters
}

\author{
A Novel SC Oscillator \\ J. L. HUERTAS, A. RODRÍGUEZ-VÁZQUEZ, \\ AND B. PÉREZ-VERDÚ
}

\section{INTRODUCTION}

Switched-capacitor (SC) networks have been recognized as an efficient tool for performing analog (sampled-data) signal processing in monolithic integrated circuit form. Such an appealing feature is a good motivation for investigating new application areas beyond the traditional simulation of analog filters. In particular, SC oscillators begin to emerge as an alternative for low- and medium-frequency sinusoidal waveform generation. Several circuit realizations for SC oscillators have been reported recently [1]-[4] and it seems interesting to search for more advantageous circuit realizations.

The purpose of this paper is to present a simple SC oscillator using unity-gain buffers and a minimum number of capacitors. When compared with the oscillator reported in [1], the new structure results more appropriate to be acommodated in a fully integrated MOS system because it does not require either additional resistors nor high capacitor ratios. On the other hand, if compared with the oscillators reported in [2]-[4], the new design does not employ infinite gain devices. Moreover, the proposed circuit is a robust oscillator since it is based on a nonlinear oscillator mechanism.

Manuscript received October 21, 1983.

The authors are with the Departamento de Electricidad y Electronica, Fac. de Física, Universidad de Sevilla, Avda Reina Mercedes s/n, Sevilla, Spain.

\section{PROPOSED OSCILlator}

Fig. 1 shows the new SC oscillator which employs two unitygain buffers. Switches appearing in that figure are controlled by a two-phase nonoverlapping clock. One phase of the clock will be named as even phase and the other as odd phase. Furthermore, we will assume a 50-percent duty cycle clock with a period of $T_{c}$ seconds. Supposing linear operation for both active devices we may formulate the following equation set:

$$
\left[\begin{array}{l}
V_{C_{1}}^{e}(n) \\
V_{C_{2}}^{e}(n)
\end{array}\right]-\left[\begin{array}{cc}
1 & C_{4} / C_{1} \\
-C_{3} / C_{2} & 1
\end{array}\right]\left[\begin{array}{l}
V_{C_{1}}^{e}(n-1) \\
V_{C_{2}}^{e}(n-1)
\end{array}\right]
$$

This linear transformation has two characteristic roots given by

$$
Z_{1,2}=1 \pm j \sqrt{C_{3} C_{4} / C_{1} C_{2}}
$$

which obviously lie outward the unity circle. This result suggests that linear modeling is inaccurate because the growing nature of the output will force the system to saturate.

Thus we need to take into consideration that at least one of the buffers must go into saturation: When that happens, both eigenvalues move towards the unit circle and reach it for some amplitude value. As it is well known, oscillation will be maintained for that amplitude. Then, for proper oscillation operation at least one of the output signals $\left(V_{01}\right.$ or $\left.V_{02}\right)$ would be saturated In order to get a low-distortion sinusoidal output we need to force that only one of the buffers is saturated during a half cycle. Moreover, we will require that the same buffer reaches saturation during both clock phases.

Let us choose $V_{01}$ as the output of the buffer going into 

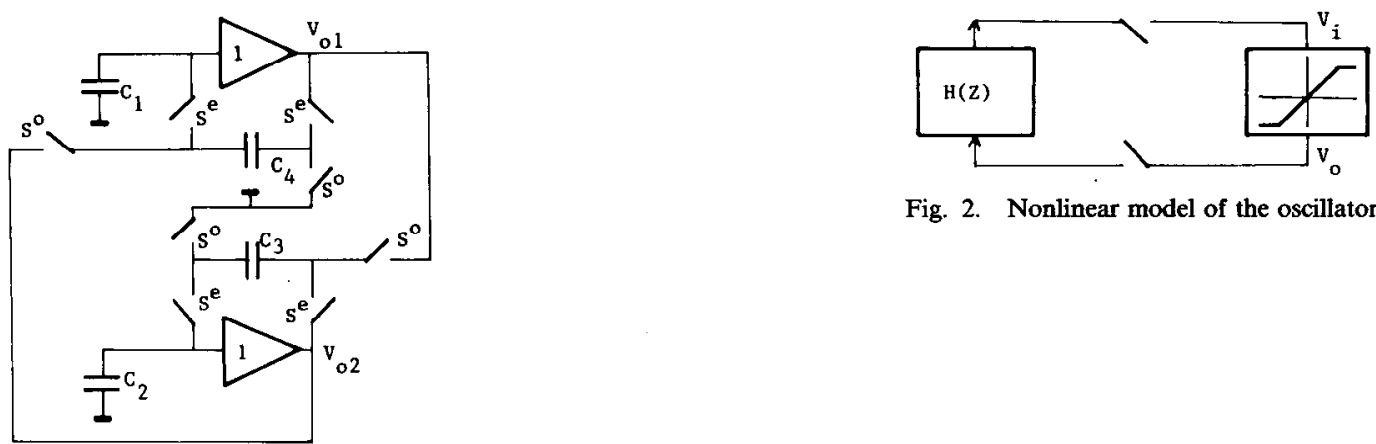

Fig. 2. Nonlinear model of the oscillator.

Fig. 1. Proposed oscillator.

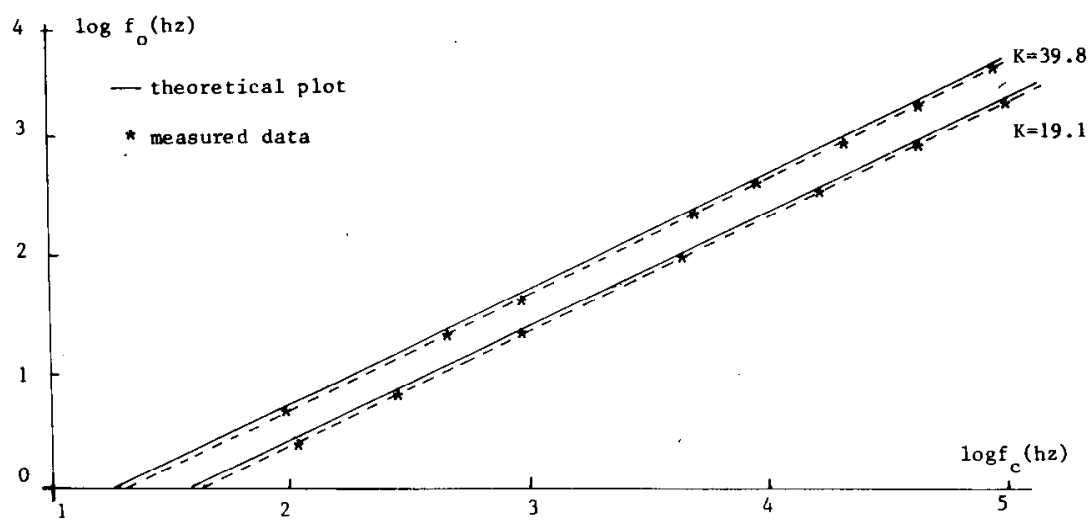

Fig. 3. Plot of oscillation frequencies against clock frequencies for two differ ent $k$ values.

saturation. It can be shown that a necessary condition which guarantees that $V_{02}$ does not reach saturation too is

$$
\frac{C_{4}}{C_{1}}>\frac{C_{3}}{C_{2}}
$$

With the above assumption in mind, we can write the state equation for the system taking into account saturation nonlinearity

$$
\begin{array}{r}
{\left[\begin{array}{c}
V_{C_{1}}^{e}(n) \\
V_{C_{2}}^{e}(n)
\end{array}\right]=\left[\begin{array}{cc}
\frac{C_{1}}{C_{1}+C_{4}} & \frac{C_{4}}{C_{1}+C_{4}} \\
0 & 1
\end{array}\right]\left[\begin{array}{c}
V_{C_{1}}^{e}(n-1) \\
V_{C_{2}}^{e}(n-1)
\end{array}\right]} \\
+\left[\begin{array}{c}
\frac{C_{4}}{C_{1}+C_{4}} \\
-C_{3} / C_{2}
\end{array}\right] f\left[V_{C_{1}}^{e}(n-1)\right]
\end{array}
$$

with

$$
f\left[B V V_{C_{1}}^{e}(n)\right]=\left\{\begin{array}{cll}
V_{C_{1}}^{e}(n), & \text { for } & -V_{\text {sat }}<V_{C_{1}}^{e}(n)<V_{\text {sat }} \\
V_{\text {sat }}, & \text { for } & V_{C_{1}}^{e}(n)>V_{\text {sat }} \\
-V_{\text {sat }}, & \text { for } & V_{C_{1}}^{e}(n)<-V_{\text {sat }}
\end{array}\right.
$$

where $V_{\text {sat }}$ is the saturation voltage for $V_{01}$ and we have assumed a symmetrical saturation for that buffer.

Finding an exact solution for (4) is rather involved. Instead, we will consider an approximate solution based on the use of a first order describing function [6]. To do that, we will model the oscillator by the block diagram shown in Fig. 2 where the linear block may be described by

$$
H(Z)=\frac{C_{4}}{C_{1}+C_{4}} \frac{Z^{2}-Z-\frac{C_{3}}{C_{2}}}{Z^{2}+\left(\frac{C_{4}}{C_{1}+C_{4}}-2\right) Z+\frac{C_{1}}{C_{1}+C_{4}}} .
$$

We will assume a sinusoidal input to the nonlinearity given by

$$
V_{i}(n)=A \sin \left(n w_{0} T_{c}\right)
$$

where $T_{c}$ is the clock period, $A$ is the oscillation amplitude, and $w_{0}$ is the angular oscillation frequency.

Now, we may represent the static nonlinearity by an equivalent gain $N(A)$ corresponding to the first harmonic of the nonlinearity output. After some algebraic manipulations we obtain the value of $f_{0}$

$$
f_{0}=\frac{f_{c}}{2 \pi} \cos ^{-1}\left[1-\frac{1}{2\left\{1+\frac{C_{1}}{C_{4}}\left(1+\frac{C_{2}}{C_{3}}\right)\right\}}\right]=\frac{f_{c}}{K} .
$$

Also, the oscillation amplitude may be evaluated from the cquation

$$
\frac{1}{1+\frac{C_{3}}{C_{2}}}=\frac{2}{\pi}\left[\sin ^{-1}\left(\frac{V_{\text {sat }}}{A}\right)+\frac{V_{\text {sat }}}{A} \sqrt{1-\left(\frac{V_{\text {sat }}}{A}\right)^{2}}\right] .
$$

\section{Discussion OF ResUlts}

Empirical data have been collected for different capacitor ratios. Agreement with theoretical predictions seems reasonable taking into account the nonexact nature of the describing function approach. In Fig. 3 we have plotted the dependance between 


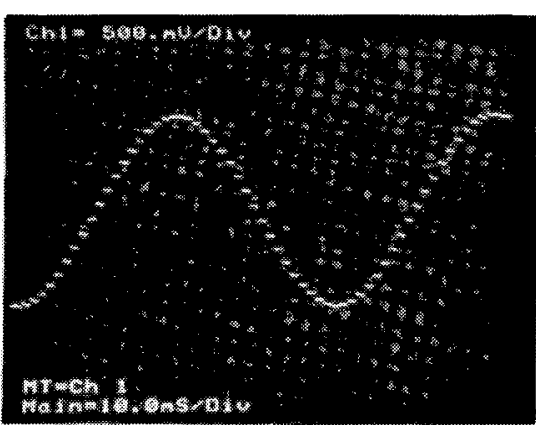

(a)

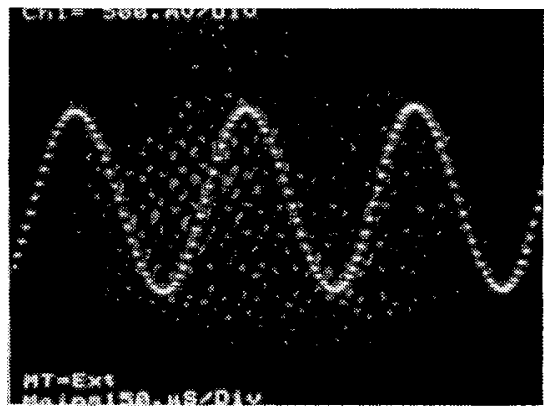

(b)

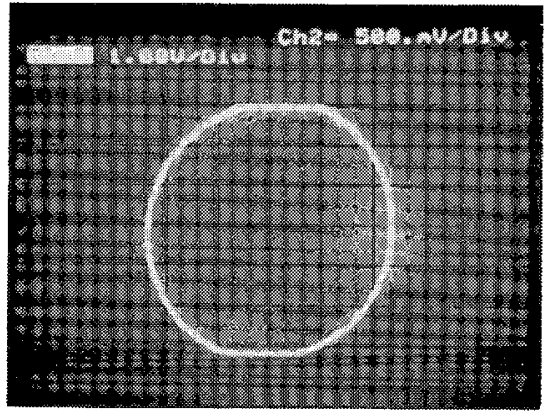

(c)

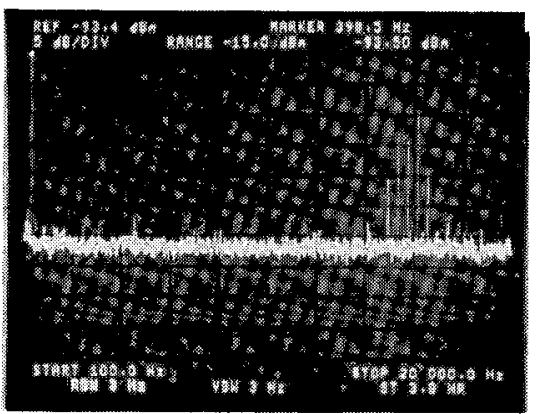

(d)

Fig. 4. (a) Output waveform for $f_{c}=669.3 \mathrm{~Hz}$; measured oscillation frequency: $17 \mathrm{~Hz}$. (b) Output waveform for $f_{c}=81229 \mathrm{~Hz}$; measured oscillation frequency: $2063 \mathrm{~Hz}$. (c) Limit cycle for $f_{c}=15690 \mathrm{~Hz}$. Horizontal axis: $V_{02}, 0.5 \mathrm{~V} /$ div. Vertical axis: $V_{01}, 1 \mathrm{~V} /$ div. (d) Measured spectra for $f_{c}=15690 \mathrm{~Hz}$.

both oscillation and clock frequencies for two different theoretical values of parameter $k$ in (8) (namely, $k=19.1$ and $k=39.8$ ). Measured $\kappa$ were 21.3 and 44.2 , respectively. From that figure, it should be clear that linearity is preserved over a wide range of clock frequency values.

Fig. 4 depicts some oscillograms showing the performance of the new circuit. We have used the same capacitor values for all the pictures in Fig. $4\left(C_{1}=5.6 n f ; C_{2}=30 n f ; C_{3}=1.8 n f ; C_{4}=\right.$ $3 n f)$. Fig. 4(a) and (b) show the output voltage $V_{02}$ for two different clock frequency values. Amplitude does not suffer a significant change because we have used the same capacitor values. Fig. 4(c) represents the form of the limit cycle for a clock frequency of $15690 \mathrm{~Hz}$. The measured oscillation frequency was 398.5 Hz. Vertical axis corresponds to $V_{01}$ and exhibits the predicted saturation. By the contrary, $V_{02}$ (horizontal axis) does not saturate. Finally, Fig. 4(d) illustrates the output signal distortion. It shows the experimental spectral analysis for the output $V_{02}$ of the oscillator whose limit cycle is shown in Fig. 4(c). As can be seen in Fig. 4(d), the only significant low-frequency component is the first harmonic. In fact, the second and third harmonics were measured to be, respectively, -41 and $-47 \mathrm{~dB}$ below the fundamental one.

Finally, it is worth considering the influence of parasitic capacitances. We note that the structure can be made insensitive to bottom capacitances. However, it will be sensitive to top ones. It means that the design equations must slightly changed in order to include stray capacitors. For the practical cases considered above we have evaluated the incidence of strays in a pesimistic situation. Assigning to the top capacitor associated with $C_{j}(j=$ $1,2,3,4)$ a valuc of the 5 percent of $C_{j}$, we got an actual frequency deviation about the nominal value ranging from 0.39 to 1.91 percent.

\section{CONCLUSIONS}

A new SC oscillator has been reported. The proposed circuit is a fully $\mathrm{SC}$ and does not require any resistor. In addition, that oscillator is robust and its oscillatory mechanism depends only on the active elements used. Finally, there exists a linear relation between both the clock and the oscillation frequencies which is preserved for three decades. It has very good performance for a low-priced components VCO.

\section{REFERENCES}

[1] T. R. Viswanathan, K. Singhal, and C. Metzer, "Application of switchedcapacitor resistors in RC oscillators," Electron. Lett., vol. 14, pp. 659-660, Sept. 1980

[2] W. B. Mikhael and S. Tu, "Switched-capacitor oscillators with linear frequency control," in Proc. ISCAS-81, pp. 188-191, 1981.

[3] ___, "Frequency compression employing switched-capacitor oscillators and its application to FM detection," in Proc. ISCAS-8I, pp'. 58-61, 1981

[4] P. V. Ananda Mohan, V. Ramachandran, and M. N. S. Swamy, "Passive and active switched-capacitor networks," in Proc. ISCAS-82, pp. 233-235. 1982.

[5] G. C. Temes, "Mos switched-capacitor filters: History and state of art," in Proc. ECCTD-81, pp. 176-185, 1981.

[6] D. P. Atherton, Stability of Nonlinear Systems. New York: Wiley, 1981.

\section{Optimum Adaptive Algorithms with Applications to Noise Cancellation}

WASFY B. MIKHAEL, FRANK WU, GEORGE KANG, AND LARRY FRANSEN

Abstract -A new simple formulation for the choice of the optimum convergence factor $\mu$ in adaptive filtering using gradient techniques is given. This leads to several optimum adaptive filtering algorithms each of

Manuscript received October 27, 1983. This work is supported by the National Science Foundation under Grant PRM8011453, and the Naval Research Laboratory under Grant SFRC \# 82 K 2047.

W. B. Mikhael and F. Wu are with the Department of Electrical Engineering, West Virginia University, Morgantown, WV 26506.

G. Kang and L. Fransen are with the Naval Research Laboratory, Washington, DC 20375 